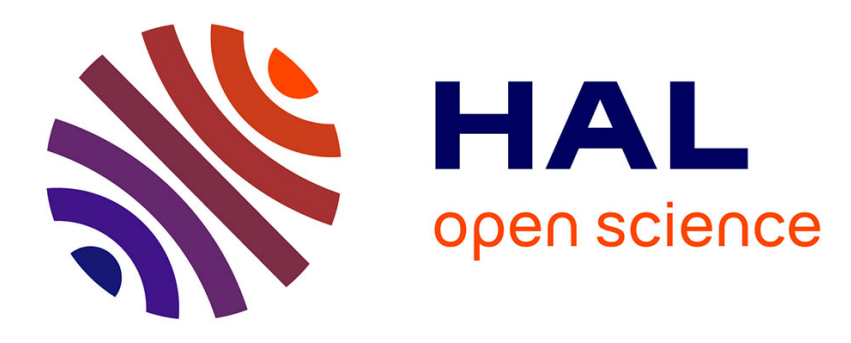

\title{
Dispatching policy for manufacturing jobs and time-delay plots
}

Nikolaos Giannelos, Nikolaos Papakostas, Dimitris Mourtzis, George

Chryssolouris

\section{- To cite this version:}

Nikolaos Giannelos, Nikolaos Papakostas, Dimitris Mourtzis, George Chryssolouris. Dispatching policy for manufacturing jobs and time-delay plots. International Journal of Computer Integrated Manufacturing, 2007, 20 (04), pp.329-337. 10.1080/09511920600786604 . hal-00513380

\section{HAL Id: hal-00513380 \\ https://hal.science/hal-00513380}

Submitted on 1 Sep 2010

HAL is a multi-disciplinary open access archive for the deposit and dissemination of scientific research documents, whether they are published or not. The documents may come from teaching and research institutions in France or abroad, or from public or private research centers.
L'archive ouverte pluridisciplinaire HAL, est destinée au dépôt et à la diffusion de documents scientifiques de niveau recherche, publiés ou non, émanant des établissements d'enseignement et de recherche français ou étrangers, des laboratoires publics ou privés. 


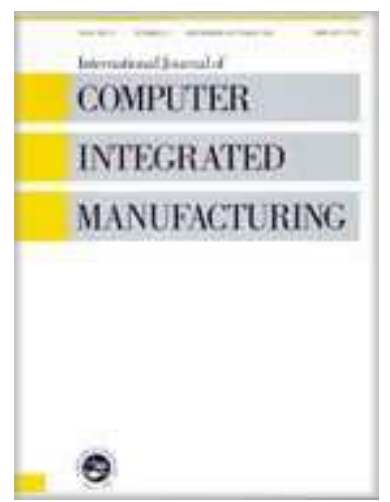

\section{Dispatching policy for manufacturing jobs and time-delay plots}

\begin{tabular}{|c|c|}
\hline Journal: & International Journal of Computer Integrated Manufacturing \\
\hline Manuscript ID: & TCIM-2005-IJCIM-0055.R2 \\
\hline Manuscript Type: & Original Manuscript \\
\hline $\begin{array}{r}\text { Date Submitted by the } \\
\text { Author: }\end{array}$ & 25-Apr-2006 \\
\hline Complete List of Authors: & $\begin{array}{l}\text { Giannelos, Nikolaos; University of Patras, Laboratory for } \\
\text { Manufacturing Systems and Automation } \\
\text { Papakostas, Nikolaos; University of Patras, Laboratory for } \\
\text { Manufacturing Systems and Automation } \\
\text { Mourtzis, Dimitris; University of Patras, Laboratory for } \\
\text { Manufacturing Systems and Automation } \\
\text { Chryssolouris, George; University of Patras, Laboratory for } \\
\text { Manufacturing Systems and Automation }\end{array}$ \\
\hline Keywords: & CONTROL, DECISION SUPPORT SYSTEMS, SCHEDULING \\
\hline Keywords (user): & \\
\hline
\end{tabular}

\section{S ScholaroNE" \\ Manuscript Central}




\title{
Dispatching policy for manufacturing jobs and time-delay plots
}

\author{
N. GIANNELOS $\dagger$, N. PAPAKOSTAS $\dagger$, D. MOURTZIS $\dagger$ and \\ G. CHRYSSOLOURIS* $\dagger$ \\ $\dagger$ Laboratory for Manufacturing Systems and Automation, \\ Department of Mechanical Engineering and Aeronautics, \\ University of Patras, Patras 265 00, Greece, \\ Tel.: +30 2610997262 , Fax: +30 2610997744 \\ *Corresponding author: Email: xrisol@lms.mech.upatras.gr
}

\begin{abstract}
In this paper, the concepts of chaos and non-linear dynamics are briefly introduced and the tools used in the characterization of a chaotic or non-linear system are discussed for studying the performance of dispatching policies in manufacturing systems. The scheduling of a simple manufacturing system with the help of common assignment rules has been simulated first. The results are studied and analysed with the help of timedelay plots. Some conclusions are drawn and, based on them, a new method of scheduling is proposed. The method is tested against conventional rules and the results are evaluated and discussed, specifically by addressing practical and generalisation issues.
\end{abstract}

Keywords: Scheduling; Control; Decision support systems

\section{Introduction}

Chaotic systems may be analysed by using the theory of systems dynamics. A plot of the state space of a system enables the view of a system's behaviour in geometric form. Phase portraits, in particular, displaying time-delayed data of specific characteristics on 
each axis, are often used for analysing the dynamic behaviour of a system. In the state space, different types of attractors may be observed: points, which the system settles on or oscillates around (Figure 1). The simplest and most stable attractor is the fixed point: all surrounding orbits are attracted to a single point of stability. Unpredictable behaviour gives rise to chaotic or strange attractors, which have more complicated forms in the state space (phase portrait).

Production systems are often characterised as complex systems (Chryssolouris 2005), exhibiting integration, interoperability and performance problems (Valckenaers et al. 2003). Nevertheless, application of the chaos theory to production systems is considered as a relatively new area (Schmitz et al. 2002). In production systems, the models considered usually involve a system of liquid tanks with a switched flow server. In such a model, fluid continuously flows out of each tank and the server can fill one tank at a time at a constant rate so as to compensate for the fluid loss. Statistical analysis was used over a model of differential equations in order to show the chaotic behaviour of such a system (Chase et al. 1993). In a strict theoretical sense, the presence of chaos in simple discrete production models has not been solidly proven (Schmitz et al. 2002). One of the commonly acclaimed characteristics of chaotic behaviour, i.e. sensitivity to initial conditions, seems to be present in real manufacturing systems. Empirical evidence on such behaviour has been reported (Beaumariage and Kempf 1994, Kempf 1996, Hilton 1998). Furthermore, possible chaotic behaviour has been studied by using non-linear dynamics theory and approaches (Scholz-Reiter et al. 2002).

Most of the research work, related to chaos and manufacturing, investigates if and how a manufacturing system exhibits chaotic characteristics. However, there are a number of tools and methods used to detect and measure chaos in a system that can be 
of interest in studying the behaviour of a manufacturing system (Chryssolouris et al. 2004). Such tools include phase portraits and a graphic representation of the phase space of a system. The phase space represents all possible combinations of all variables of a system. Therefore, the dimension of a phase space depends on the number of the system's variables. According to (Takens 1981) and (Parunak 1995), such plots capture the topology of the system to which there is no direct access.

A phase portrait is constructed by plotting all combinations of a system's variables, as the system evolves through time. In most cases, these combinations are a subset of all possible combinations, since the laws, governing a system, exclude certain combinations of values. The resulting phase portrait allows for an examination of the system's evolution, regardless of the time flow. More specifically, the phase portrait depicts the system's succession of states (variables combinations), despite their sequence. As an example, the phase portrait of a simple pendulum is examined, as depicted in Figure 1.

[Insert figure 1 about here]

In this phase portrait, the system's pairs of variables are plotted: the speed of the pendulum (plotted on the y-axis) and the displacement of the equilibrium point (plotted on the $\mathrm{x}$-axis). The resulting phase portrait is a circle: the maximum speed is measured at the equilibrium point, as the pendulum passes this point from either side, while the speed is equal to zero at the maximum displacement points.

Each point of this phase portrait represents the complete state of knowledge about the pendulum at a single instant in time. The next point represents the state of the 
system at the next instant and so on. However, since the system is deterministic, i.e. its variables change according to solid physical laws, the essence of time is diminished in the phase portrait. What an observer can see though in a phase portrait, is a series of the system's state changes that may happen at any given time, but will invariably follow the trajectory indicated by the points in the diagram. A deterministic system, which would be the same in future as it had been in the past, would be represented by a periodical trajectory in the phase space, returning to the point representing the past state, and from then on passing through all subsequent points, ending back to the point representing the future state, exactly as shown in the pendulum example of Figure 1.

Similarly, when the trajectory of a phase space doesn't enter a certain area, the values the variables of the system would have in that area are excluded. Thus, by studying a phase portrait, loops, periodicities, or impossible states of a system may be identified.

Summarizing, through the use of phase portraits, the state of a physical system at an instant, is represented as one point in the phase space. As the system changes and when the variables of the system never become infinite, the map of the points will be outlined in a bounded part of the entire phase space.

Some systems converge to some pattern of values, which is called an 'attractor' for the system. An attractor 'attracts' the points around it in the phase space. The points surrounding each attractor constitute the 'basin of attraction'. When the state of a system is represented by a point within the basin of attraction, the system will eventually follow the dynamics of the corresponding attractor. In case the system gets into a different basin of attraction, the system will finally converge to that specific basin's attractor. In order to represent an attractor of a chaotic system, a stable, non- 
periodic, bounded pattern is required, where the paths leading to nearby points would rapidly diverge. The chaotic pattern requires, therefore, an infinite series of points (since it never passes from the same point twice) in a finite area. This pattern is called a 'strange attractor.' A quite widely known type of such attractors is the Lorenz attractor (Lorenz 1963), a three dimensional body that may be seen in Figure 2.

[Insert figure 2 about here]

The work reported in this paper, focuses on the use of time-delay plots, a particular type of phase portraits, for studying the behaviour of dispatching rules in a manufacturing system and for devising a different dispatching policy, based on the analysis of the time-delay plots.

\section{Dispatching rules and phase portraits}

In order for the behaviour of different dispatching policies to be examined with the help of time-delay plots, two sets of experiments were designed and executed, using three dispatching rules applied to two simple machine models:

- $\quad$ Shortest Processing Time (SPT).

- First-Come-First-Serve (FCFS).

- Earliest Of Due Dates (EODD).

The performance of these rules was evaluated with the use of four performance indicators, namely:

- Mean Tardiness, which, for each job, is given by the following equation: 
$T_{n}=\max \left[0, D D_{n}-E T_{n}\right]$, where $T_{n}, E T_{n}$ and $D D_{n}$ represent the tardiness, the completion (end) time and the due date of job $n$, respectively.

- Mean Lateness, which, for each job, is given by the following equation: $L_{n}=E T_{n}-D D_{n}$

- Mean Flowtime, which, for each job, is given by the following equation: $F_{n}=E T_{n}-A T_{n}$, where $A T_{n}$ is the arrival time of job $n$, and

- Fraction Tardy, which is the fraction of delayed jobs, expressed as percentage.

The first set of experiments was conducted by dispatching a string of 5000 jobs in a single resource according to each rule. The 5000 jobs were described with the use of three variables (arrival time, processing time and due date). The arrival times were randomly generated, following an exponential distribution. The mean inter-arrival time was assigned the values of 250, 125 and 62.5, representing rare, frequent and very frequent arrivals respectively. The processing times were generated having followed a normal distribution with a mean value of 100 and a standard deviation that varied among the values 10, 20 and 30. Zero and/or negative processing times were not allowed. Finally, the due dates were calculated having followed a uniform distribution of random values within the range [arrival time, arrival time + PTA], where PTA is the processing time average of all the jobs in the string. For each set of variables governing the distribution of arrival times, processing times and due dates, the experiment was conducted 20 times (i.e. 20 strings of 5000 jobs each were assigned to the resource) and the mean values of the performance indicators were calculated. 
In the following Tables 1, 2 and 3, we present the performance results of the three scheduling rules for a standard deviation of 20 and for inter-arrival time equal to 250 , 125 and 62.5 respectively.

[Insert tables 1, 2 and 3 about here]

The values presented in these tables are very close to the ones for a standard deviation of 10 or 30 , since the standard deviation of the mean processing time seems to be having the least effect on the performance of the dispatching rules.

Furthermore, a series of experiments was conducted, during which the processing time for each job was assigned a value from a uniform distribution (instead of a normal one) within the boundaries [5, 105]. The due dates for this series of experiments was fixed to be 20,50 or 100 time units after the arrival time. The mean inter-arrival time was kept at 125 time units. The performance of rules for these sets of values may be seen in the following Tables 4,5 and 6 .

[Insert tables 4, 5 and 6 about here]

For this set of experiments, the following conclusions may be drawn:

1. FCFS and EODD are producing identical results, owing to the fact that the due date is always a predefined period of time after the arrival time, and

2. Mean flowtime is always equal to mean lateness plus the amount of time units used to calculate the due date for the same reason as above. 
A time-delay plot may be developed for each rule, based on the job flowtime variable (time for a job to flow through the system). This kind of diagrams may easily be constructed by plotting each value of a data series on the Y-axis (abscissa) against the previous value on the $\mathrm{X}$-axis (ordinate). As an example, the coordinates of the first two points of the plot are given by $\left(\mathrm{F}_{1}, \mathrm{~F}_{2}\right)$ and $\left(\mathrm{F}_{2}, \mathrm{~F}_{3}\right)$, respectively. By examining these plots, defining characteristics of each rule may come to light. Should this be possible, the characteristics of the time-delay plot, corresponding to the best rule, as determined previously, may be used to develop a new procedure in order for an even better performance in terms of the performance indicators to be achieved.

For each scheduling rule, a time-delay plot is constructed, displaying the flowtime of job $n$ versus the flowtime of job $n+1$, for the entire string of 5000 jobs, in the order they are dispatched (Figure 3, Figure 4, Figure 5).

[Insert figures 3, 4 and 5 about here]

The observation of the time-delay plots reveals an interesting characteristic: there appears to be some orderly structure in the SPT phase portrait, while the FCFS and EODD diagrams demonstrate a strong diagonal band of points. Furthermore, the FCFS time-delay plot seems to be a subset of the EODD, with a diagonal line marking the limit between the two plots.

This may indicate that SPT strongly affects the way flowtime is distributed among jobs. On the contrary, EODD and FCFS time-delay plots show rather irregular patterns, indicating that these rules do not directly affect the distribution of flowtime among jobs. 
A second observation can be made by examining the way these time-delay plots evolve as the workloads are becoming denser (i.e. more often arrivals and larger standard deviations). The time-delay plots presented above, correspond to a mean interarrival of 125. In the following Figures 6 and 7, we can see the time-delay plots of FCFS and SPT for a mean inter-arrival of 62.5 (EODD plots for these values are almost identical to FCFS). The lines connecting successive points have been removed from these diagrams in order to facilitate the observation of the points being spread across the plane.

[Insert figures 6 and 7 about here]

Apparently, FCFS and EODD have a tendency to produce time-delay plots with all the points being located very closely to the diagonal (meaning that the flowtimes of all the jobs follow a regular pattern, without large deviations), while SPT tends to spread the points far away from the diagonal. Consequently, SPT tends to produce some extremely large flowtimes but keeps most of them quite low, thus, resulting in a good performance. On the other hand, FCFS and EODD keep the flowtime of each job closer to the mean flowtime value, resulting not only in much lower maximum values, but also in producing a poor performance.

\section{Time-delay plot-based rule}

Based on the observations of the previous section, a new scheduling rule can be devised. Since SPT seems to be producing better results, whilst at the same time is spreading the 
flowtimes away from the diagonal, an argument can be made as to whether by spreading the flowtimes, satisfactory results may be obtained.

However, it turns out that the task of spreading out the points in a time-delay plot is "easy". Obvious solutions (e.g. select the task that will result into plotting a point as far away as possible from the diagonal) tend to actually produce worse results, since after a small number of assignments, all the remaining alternatives tend to be on the diagonal. After having experimented with various rules, it was discovered that the one tending to scatter the points of the time-delay plot most, is the following:

At each decision point, from all the available jobs, select the one which, if executed, will have a flowtime as close as possible to the average processing time of all the jobs that have arrived so far.

[Insert figure 8 about here]

The new rule (Time-Delay plot Rule - TDR) was tested, along the three already mentioned rules, against new sets of values for the workloads. In Figure 9, the timedelay plot of the rule (again, connecting lines have been omitted) is shown for a mean inter-arrival time of 83.3 , mean process time 120 and a standard deviation of 20 , for a single machine. The performance of all the rules for this set of values is demonstrated in Table 7. Other sets of values have produced similar results.

[Insert table 7 about here] 
By observing the time-delay plot in Figure 8 , it is obvious that there are still many points very close to the diagonal, but there are more on either side of it. As expected, this rule behaves quite differently from the three rules already tested. Its results tend to be somewhere between SPT on the one side and EODD and FCFS on the other. One could expect that another rule, with greater success in spreading the points of its time-delay plot, would have even better results. Further investigation on this issue may be needed.

To test the proposed rule under moderate workload conditions, a second set of values was used: the mean inter-arrival time is 150 , the mean process time 120 with a standard deviation of 20 , while the due dates were fixed to be 300 time units after the arrival time. It is, therefore, expected that EDD and FCFS will behave similarly here.

\section{[Insert table 8 about here]}

Apparently, in the last experiment, the proposed rule seems to have worse performance in terms of tardiness but achieves the best fraction tardy performance of all rules used. Having tested the performance of the proposed rule in a quite extended series of other experiments, it seems that the rule performs better under heavy workloads with tightly fixed due dates.

One of the most important advantages of the underlying approach is that timedelay plots may easily be constructed for studying the behaviour and performance of real manufacturing systems. Specifically, time-delay plots may be created for a specific resource, workcenter or an entire factory: if the appropriate data is available from any existing data vault, such as a database, the patterns of change (in time) of the values of a 
series of performance indicators along with the minimum / maximum values and corresponding distribution may well be analysed and compared with previous patterns recorded in the past or with patterns belonging to other resources, workcenters or even factories. Parts of these plots may be isolated for studying the behaviour of the system in a specific time frame. Furthermore, this kind of data representations may also be used for revealing trends and relationships that are not apparent or easily detectable, thus having a potentially useful role to play in the context of data mining projects within manufacturing environments.

\section{Results and discussion}

The use of chaos-related concepts, such as phase portraits and time-delay plots, reveal interesting geometric patterns for the variables, associated with the production scheduling problem, and may often bring to light orderly structures. Similar analysis of other scheduling variables or interrelationships between variables, e.g. flowtime and tardiness, may also reveal orderly patterns.

The use of phase portraits and time-delay plots to test other performance measures in place of flowtime, such as fraction tardy, tardiness, lateness, completion time, and waiting time may also be taken into consideration for future research.

The implications of the work described in this paper can be far-reaching in the sense that, after adequate experimentation, it may be possible to create a "dictionary", of various attractors or patterns in the phase portraits or time-delay plots, corresponding to different scheduling rules. One may then be able to choose the performance measure most desired and to create a schedule that will correspond to the attractor in the phase portrait or time-delay plot. Pattern recognition models may be developed for analysing 
behaviour and for identifying underlying trends and rules for improving the performance of a manufacturing system. Furthermore, geometric manifestations of nonlinear dynamics, such as fractals, along with their mathematical characteristics, such as similarity, Hausdorff and correlation dimensions, may be used for capturing the inner characteristics of a manufacturing system. Admittedly, both the experiment and methodology developed here are relatively small-scaled; they may open up, however, possibilities for future work.

\section{References}

[1] Beaumariage, T. and Kempf, K., The Nature and Origin of Chaos in Manufacturing Systems, Proceedings of the IEEE/SEMI Advanced Semiconductor Manufacturing Conference, 1994, 169-174.

[2] Chase, J., Serano, J. and Ramadge, P.J., Periodicity and Chaos from Switched Flow Systems: Contrasting Examples of Discretely Controlled Continuous Systems, IEEE Transactions on Automatic Control, 1993, 38(1), 70-83.

[3] Chryssolouris, G., Giannelos, N., Papakostas N. and Mourtzis, D., Chaos Theory in Production Scheduling, Annals of the CIRP, 2004, 53(1), 381-383.

[4] Chryssolouris, G., Manufacturing Systems - Theory and Practice, $2^{\text {nd }}$ edition, 2005 (Springer - Verlag).

[5] Hilton, C., Manufacturing Operations System Design and Analysis, Intel Technology Journal, 1998, $4^{\text {th }}$ Quarter 1998.

[6] Kempf, K.G., 1996, Simulating Semiconductor Manufacturing Systems: Successes, Failures, and Deep Questions, Proceedings of the $28^{\text {th }}$ Conference on Winter Simulation (ACM Press: NY). 
[7] Lorenz, E.N., Deterministic Nonperiodic Flow, Journal of Atmospheric Sciences, 1963, 20, 130-141.

[8] Parunak, H.V.D., The Heartbeat of the Factory, Technical Report, ERIM, Ann Arbor, www.erim.org/ vparunak/heartbt.pdf, 1995.

[9] Schmitz, J.P.M., van Beek, D.A. and Rooda, J.E., 2002, Chaos in Discrete Production Systems?, Journal of Manufacturing Systems, 2002, 21(3), 236-246.

[10] Scholz-Reiter, B., Freitag, M. and Schmieder, A., 2002, Modelling and Control of Production Systems based on Nonlinear Dynamics Theory, Annals of the CIRP, 2002, 51(1), 375-378.

[11] Takens, F., Detecting Strange Attractors in Turbulence, Dynamical Systems and Turbulence (Eds: Rand, D.A., Young, L.-S.), 1981, 898, 366-381 (Springer).

[12] Valckenaers, P., Van Brussel, H., Hadeli, Bochmann, O., Saint Germain, B. and Zamfirescu, C., 2003, On the design of emergent systems: an investigation of integration and interoperability issues, Emerging Applications of Artificial Intelligence, 16, 377-393 
URL: http://mc.manuscriptcentral.com/tandf/tcim Email:ijcim@bath.ac.uk 


\section{List of figures}

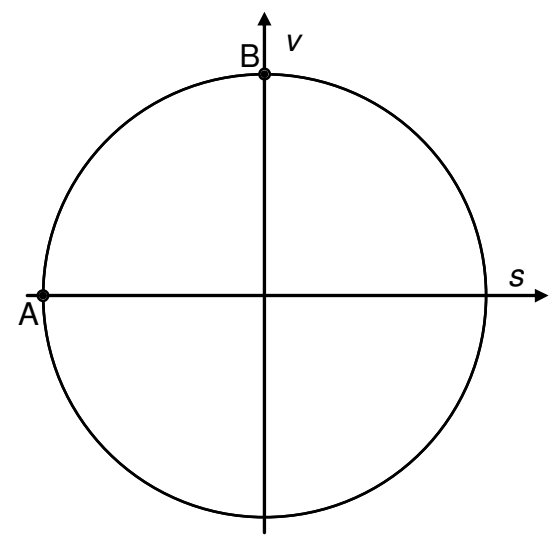

Figure 1: Phase portrait of a pendulum

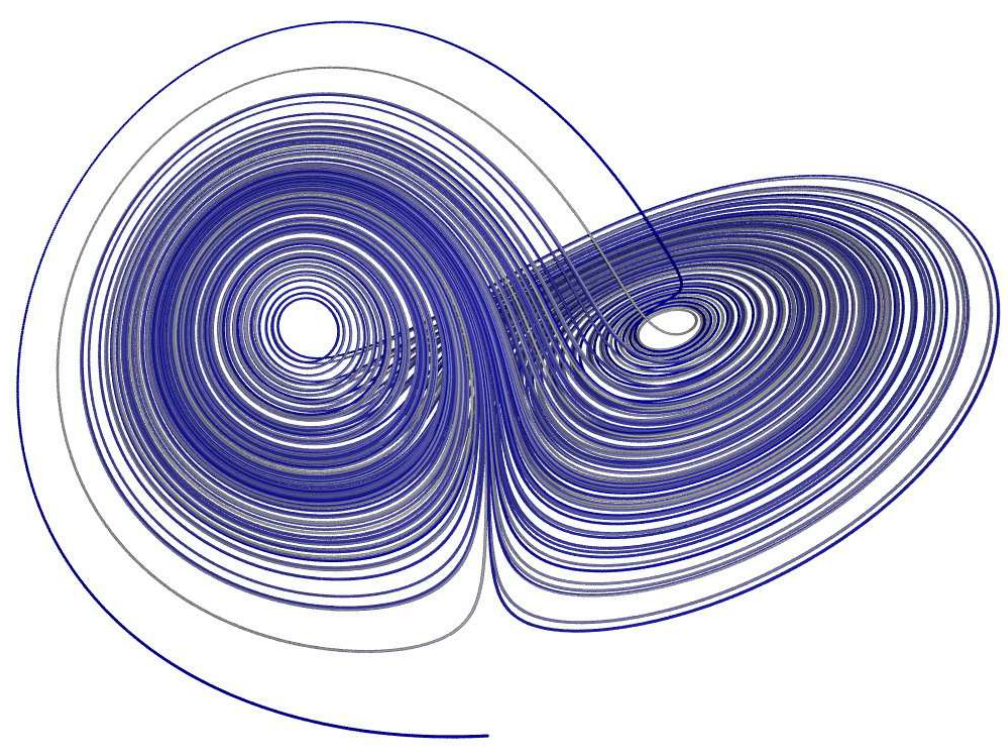

Figure 2: The Lorentz attractor 


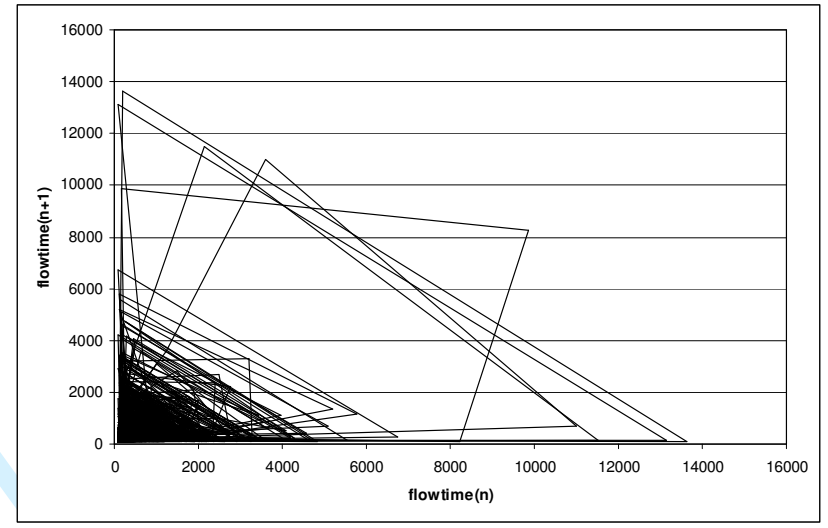

Figure 3: SPT time-delay plot.

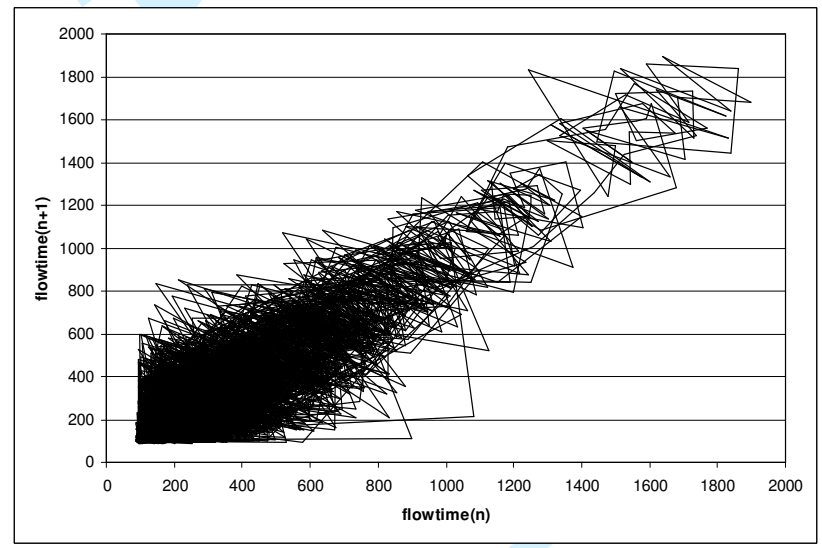

Figure 4: EODD time-delay plot.

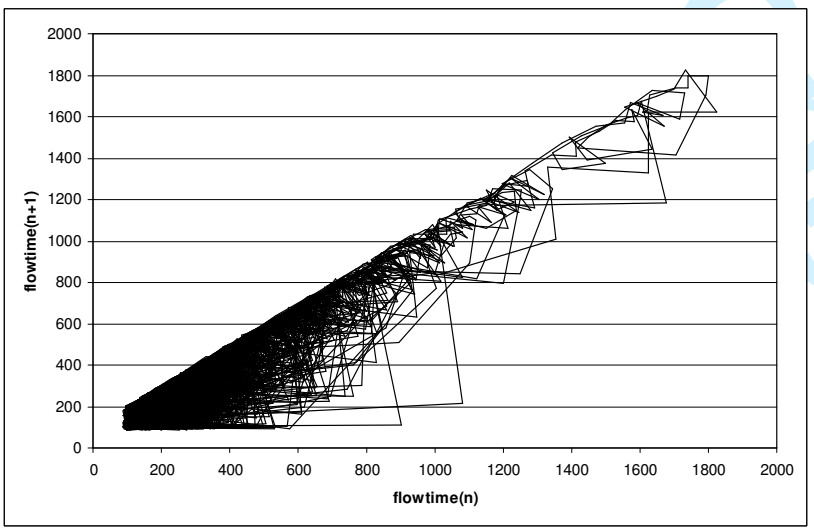

Figure 5: FCFS time-delay plot. 


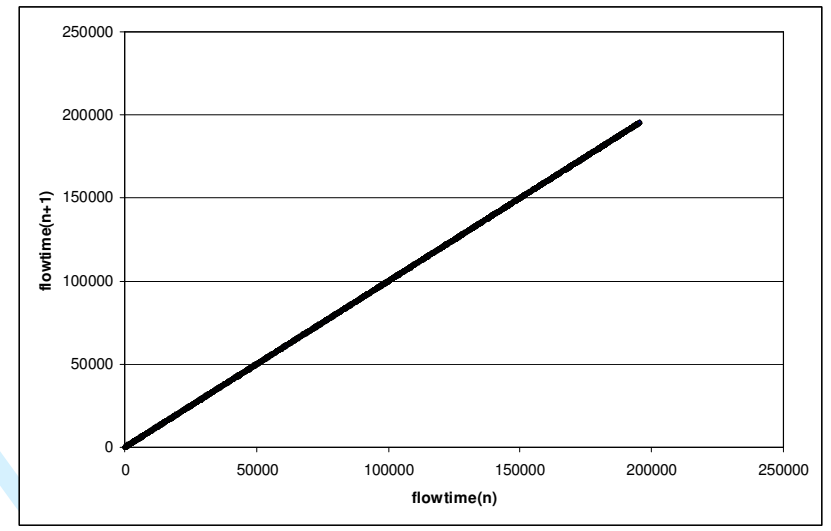

Figure 6: FCFS time-delay plot for tight workload.

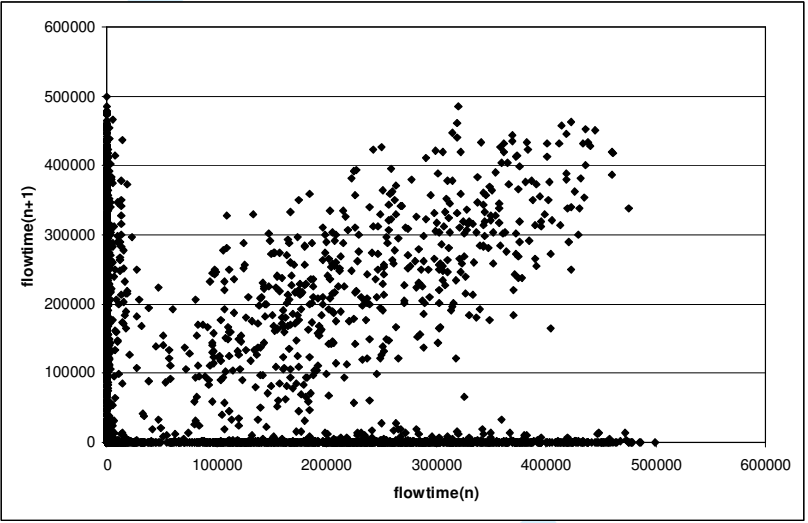

Figure 7: SPT time-delay plot for tight workload.

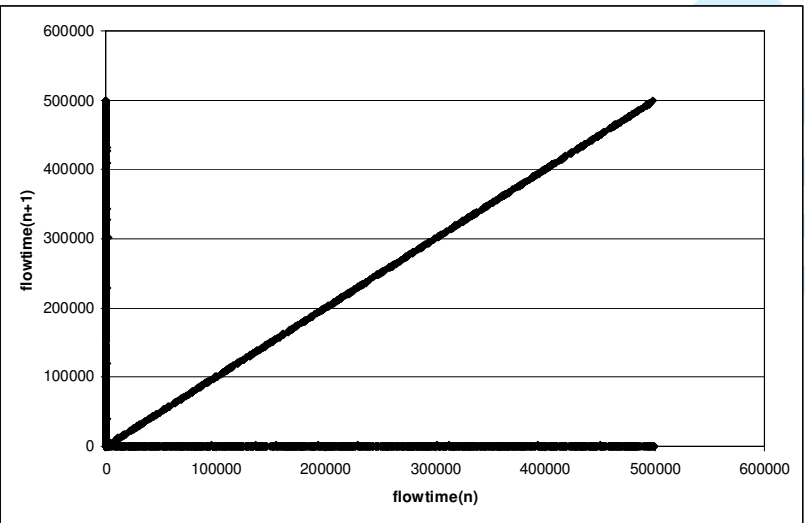

Figure 8: Time-delay plot for the proposed dispatching method. 


\section{List of tables}

\begin{tabular}{|l|l|l|l|l|}
\hline \multirow{2}{*}{ Rule } & $\begin{array}{l}\text { Mean } \\
\text { Tardiness }\end{array}$ & Lateness & Flowtime & Tardy \\
\hline SPT & 83.23 & 82.60 & 132.82 & $94.88 \%$ \\
\hline FCFS & 85.13 & 84.52 & 134.74 & $95.03 \%$ \\
\hline EODD & 85.13 & 84.53 & 134.75 & $95.07 \%$ \\
\hline
\end{tabular}

Table 1: Results of scheduling experiments for inter-arrival time 250

\begin{tabular}{|l|l|l|l|l|}
\hline \multirow{2}{*}{ Rule } & Mean & Mean & Mean & Fraction \\
& Tardiness & Lateness & Flowtime & Tardy \\
\hline SPT & 228.65 & 228.37 & 278.44 & $97.63 \%$ \\
\hline FCFS & 261.21 & 260.99 & 311.06 & $98.19 \%$ \\
\hline EODD & 261.22 & 261.00 & 311.07 & $98.22 \%$ \\
\hline
\end{tabular}

Table 2: Results of scheduling experiments for inter-arrival time 125

\begin{tabular}{|l|l|l|l|l|}
\hline Rule & Mean & Mean & Mean & Fraction \\
& Tardiness & Lateness & Flowtime & Tardy \\
\hline SPT & 75529.65 & 75529.55 & 75579.62 & $99.03 \%$ \\
\hline FCFS & 94338.92 & 94338.92 & 94388.99 & $99.99 \%$ \\
\hline EODD & 94338.92 & 94338.92 & 94388.99 & $99.99 \%$ \\
\hline
\end{tabular}

Table 3: Results of scheduling experiments for inter-arrival time 62.5 


\begin{tabular}{|l|l|l|l|l|}
\hline \multirow{2}{*}{ Rule } & $\begin{array}{l}\text { Mean } \\
\text { Tardiness }\end{array}$ & Lateness & Flowtime & Tardy \\
\hline SPT & 59.47 & 58.81 & 78.81 & $90.76 \%$ \\
\hline FCFS & 63.77 & 63.13 & 83.13 & $91.16 \%$ \\
\hline EODD & 63.77 & 63.13 & 83.13 & $91.16 \%$ \\
\hline
\end{tabular}

Table 4: Results of scheduling experiments for uniformly distributed processing times and due date equalling arrival time plus 20 time units

\begin{tabular}{|l|l|l|l|l|}
\hline \multirow{2}{*}{ Rule } & $\begin{array}{l}\text { Mean } \\
\text { Tardiness }\end{array}$ & Lean & Mean & Fraction \\
\hline SPT & 35.29 & 28.45 & 78.45 & $67.63 \%$ \\
\hline FCFS & 39.16 & 32.75 & 82.75 & $70.14 \%$ \\
\hline EODD & 39.16 & 32.75 & 82.75 & $70.14 \%$ \\
\hline
\end{tabular}

Table 5: Results of scheduling experiments for uniformly distributed processing times and due date equalling arrival time plus 50 time units

\begin{tabular}{|l|l|l|l|l|}
\hline Rule & Mean & Mean & Mean & Fraction \\
& Tardiness & Lateness & Flowtime & Tardy \\
\hline SPT & 12.47 & -21.33 & 78.66 & $23.34 \%$ \\
\hline FCFS & 14.27 & -17.03 & 82.97 & $28.46 \%$ \\
\hline EODD & 14.27 & -17.03 & 82.97 & $28.46 \%$ \\
\hline
\end{tabular}

Table 6: of scheduling experiments for uniformly distributed processing times and due date equalling arrival time plus 100 time units 


\begin{tabular}{|l|l|l|l|l|}
\hline \multirow{2}{*}{ Rule } & Mean & Mean & Mean & Fraction \\
& Tardiness & Lateness & Flowtime & Tardy \\
\hline TDR & 88863.15 & 88863.10 & 88923.13 & $99.45 \%$ \\
\hline SPT & 75621.68 & 75621.61 & 75681.64 & $99.35 \%$ \\
\hline FCFS & 92413.48 & 92413.47 & 92473.50 & $99.99 \%$ \\
\hline EODD & 92413.47 & 92413.47 & 92473.50 & $99.99 \%$ \\
\hline
\end{tabular}

Table 7: Results of scheduling experiments for each rule (tight workload)

\begin{tabular}{|l|l|l|l|l|}
\hline Rule & Mean & Mean & Mean & Fraction \\
\hline TDR & 774.92 & 61.45 & 361.45 & $21.74 \%$ \\
\hline SPT & 555.79 & 36.84 & 336.85 & $24.47 \%$ \\
\hline $\begin{array}{l}\text { FCFS \& } \\
\text { EODD }\end{array}$ & 290.23 & 69.94 & 369.94 & $46.60 \%$ \\
\hline
\end{tabular}

Table 8: Results of scheduling experiments for each rule (moderate workload) 